\title{
DISPERSIVE AND SUPERADDITIVE ORDERING
}

\author{
A. N. AHMED, ${ }^{*}$ King Saud University \\ A. ALZAID, ${ }^{*}$ King Saud University \\ J. BARTOSZEWICZ, ${ }^{* *}$ University of Wrocław \\ S. C. KOCHAR, ${ }^{* * *}$ Panjab University
}

\begin{abstract}
Recently many authors have established connections between dispersive ordering and some other partial orderings of distributions. This paper presents the connection which superadditive ordering has with dispersive ordering.
\end{abstract}

\section{Introduction}

Let $F$ and $G$ be two distribution functions and let $F^{-1}$ and $G^{-1}$ be their corresponding left-continuous inverses. $G$ is said to be more dispersed than $F$ (Lewis and Thompson (1981), written $F \stackrel{\text { disp }}{<} G$, if

$$
G\left(G^{-1}(\alpha)+a\right) \leqq F\left(F^{-1}(\alpha)+a\right) \text { for all } a>0 \text { and } \alpha \in(0,1) .
$$

Deshpande and Kochar (1983) have shown that (1.1) is equivalent to

$$
G^{-1}(\beta)-G^{-1}(\alpha) \geqq F^{-1}(\beta)-F^{-1}(\alpha) \text { whenever } 0<\alpha<\beta<1 \text {. }
$$

The last inequality is equivalent to saying

$$
G^{-1} F(x)-x \text { is non-decreasing in } x,
$$

(see for example Shaked (1982)).

The support of $F$ will be denoted by $S_{F}$. In reliability theory, if $F$ and $G$ are such that $F(0)=G(0)=0$ and $G$ is strictly increasing on $S_{G}$, an interval, three well-known orderings of distributions are introduced (see Barlow and Proschan (1975)). $F$ is said to be convex ordered with respect to $G$, written $\stackrel{c}{<} G$, if $G^{-1} F(x)$ is a convex function in $x$ on $S_{F}$, assumed an interval.

$F$ is star-ordered with respect to $G$, written $F \stackrel{*}{<} G$, if $G^{-1} F(x)$ is star-shaped, i.e., $G^{-1} F(x) / x$ is increasing in $x$ for $x \in S_{F}$.

This letter is a combined and revised version of three letters submitted in February 1986 by Ahmed and Alzaid, Bartoszewicz, and Kochar. Revised version received 4 September 1986.

* Postal address: Department of Statistics, King Saud University, PO Box 2455, Riyadh 11451, Saudi Arabia.

${ }^{* *}$ Postal address: Mathematical Institute, University of Wroclaw, Pl. Grunwaldski 2/4, 50-384 Wroclaw, Poland.

*** Postal address: Department of Statistics, Panjab University, Chandigarh 160014, India. 

if

$F$ is said to be superadditive (subadditive) with respect to $G$, written $F \stackrel{\text { su }}{<} G(F<$ su $G)$,

$$
G^{-1} F(x+y) \geqq(\leqq) G^{-1} F(x)+G^{-1} F(y) \text { for all } x, y \in S_{F} .
$$

Recently, many connections have been established among the convex ordering, the star-shaped ordering and the dispersive ordering. See Barlow and Proschan (1975), Deshpande and Kochar (1983), Sathe (1984), and Bartoszewicz (1985a), (1985b), among others.

The purpose of this paper is to establish connections between the superadditive ordering and the dispersive ordering of distributions. To avoid technical complications in the statements of the results and in the proofs we shall assume throughout that the supports of the underlying distributions are intervals and that the distributions have no atoms. Thus, these distributions will have strictly increasing and continuous inverses on $(0,1)$.

\section{The main results}

We first show that superadditive ordering neither implies nor is implied by the dispersive ordering.

Example 2.1. Let $F(x)=G(x+\theta), \theta>0$. Then $F \stackrel{\text { disp }}{<} G$ but $F \stackrel{\text { su }}{\leftarrow} G$. That is, the dispersive ordering does not imply the superadditive ordering.

Example 2.2. Let $F$ have the Weibull distribution function

$$
F(x)=1-\exp \left(-x^{2}\right), \quad x>0, \quad \text { and let } G(x)=1-\exp (-x), \quad>0 .
$$

Since $G^{-1} F(x)-x=x^{2}-x$ is not non-decreasing in $x$ for all $x \geqq 0$, then $F \stackrel{\text { disp }}{\leftarrow} G$. However, it is easy to show that $G^{-1} F$ is superadditive.

Next we establish, through the following set of results, connections between the dispersive and the superadditive orderings.

Theorem 2.3. If $F \stackrel{\text { su }}{<} G$ and $F \stackrel{\text { st }}{<} G$, then $F \stackrel{\text { disp }}{<} G$.

Proof. Assume $F \stackrel{\text { su }}{<} G$. Then

$$
G^{-1} F(x+y) \geqq G^{-1} F(x)+G^{-1} F(y) \text { for all } x, y \in S_{F} .
$$

Since $F \stackrel{\text { st }}{<} G$ implies $G^{-1} F(x) \geqq x$, then by (2.1),

$$
G^{-1} F(x+y) \geqq G^{-1} F(y)+x, \quad x, y \in S_{F} .
$$

Equivalently,

$$
G^{-1} F(x+y)-(x+y) \geqq G^{-1} F(y)-y, \quad x, y \in S_{F},
$$

which holds if and only if $G^{-1} F(x)-x$ is non-decreasing in $x \in S_{F}$, i.e., if and only if $F \stackrel{\text { disp }}{<} G$.

Denote by

$$
\bar{F}(x \mid t)=\frac{1-F(x+t)}{1-F(t)}, \quad x \geqq 0, \quad t \geqq 0,
$$

the conditional reliability of a unit of age $t$ if $F$ is the life distribution of the unit.

The following is an immediate consequence of Theorem 2.3. 
Corollary 2.4. If $\bar{F}(x \mid t) \leqq \bar{G}(x \mid t)$ for all $x \geqq 0$ and $t \geqq 0$ and $F \stackrel{\text { su }}{<} G$, then $F \stackrel{\text { disp }}{<} G$.

Remark 1. Theorem 2.3 can be used to improve Bartoszewicz's (1985a) result which says that if $F \stackrel{\text { c }}{<} G$ and $F \stackrel{\text { st }}{<} G$ then $F \stackrel{\text { disp }}{<} G$, and Bartoszewicz's (1985b) observation which states that if $F \stackrel{*}{<} G$ and $F \stackrel{\text { st }}{<} G$ then $F \stackrel{\text { disp }}{<} G$. This is so since it is well known that $F \stackrel{\text { c }}{<} G \rightarrow F \stackrel{*}{<} G \rightarrow F \stackrel{\text { su }}{<} G$ (see Barlow and Proschan (1975), pp. 107 and 109).

Sathe (1984) has pointed out that if $\lim _{x \rightarrow 0+}\left(G^{-1} F(x) / x\right) \geqq 1$ and $F \stackrel{*}{<} G$, then $F \stackrel{\text { disp }}{<} G$. In our next result, it is shown that the limit condition arises naturally under the superadditive ordering and that Sathe's conclusion still holds under the weaker condition $F \stackrel{\text { su }}{<} G$. This is contained in the following.

Lemma 2.5. If $F \stackrel{\text { su }}{<} G$, then $(d / d x) G^{-1} F(x) \geqq \lim _{y \rightarrow 0+}\left(G^{-1} F(y) / y\right)$ for all $x \in S_{F}$.

Proof. Note that as $F$ su $G$, then by (1.4),

$$
G^{-1} F(x+y) \geqq G^{-1} F(x)+G^{-1} F(y) \text { for all } x, y \in S_{F},
$$

so that

$$
\frac{G^{-1} F(x+y)-G^{-1} F(x)}{y} \geqq \frac{G^{-1} F(y)}{y} .
$$

Taking limits of both sides of (2.3) as $y \rightarrow 0$, the conclusion of the lemma follows.

Theorem 2.6. If $\lim _{x \rightarrow 0+}\left(G^{-1} F(x) / x\right) \geqq 1$ and $F \stackrel{\text { su }}{<} G$, then $F \stackrel{\text { disp }}{<} G$.

Proof. We claim that, under the assumptions of the proposition

$$
\frac{d}{d x}\left\{G^{-1} F(x)-x\right\} \text { is non-negative for all } x \in S_{F} .
$$

To see it, note that, as $F \stackrel{\text { su }}{<} G$ and $\lim _{x \rightarrow 0+}\left(G^{-1} F(x) / x\right) \geqq 1$, then by Lemma 2.5 ,

$$
\frac{d}{d x} G^{-1} F(x) \geqq 1 \quad \text { whenever } \quad x \in S_{F} \text {. }
$$

It follows that $G^{-1} F(x)-x$ is non-decreasing in $x$ for all $x \in S_{F}$. Hence by (1.3), $F \stackrel{\text { disp }}{<} G$.

Remark 2. As a byproduct of Theorem 2.6 we have the following improved version of Desphande and Kochar's (1983) observation: If $F$ and $G$ are absolutely continuous with $F(0)=G(0)=0$, and their corresponding densities are such that $f(0) \geqq g(0)>0$, then $F \stackrel{\text { su }}{<} G$ implies $F \stackrel{\text { disp }}{<} G$.

\section{References}

Barlow, R. and Proschan, F. (1975) Statistical Theory of Reliability and Life Testing. Holt, Rinehart and Winston, New York. 
Bartoszewicz, J. (1985a) Moment inequalities for order statistics from ordered families of distributions. Metrika 32, 383-389.

BARToszewiCZ, J. (1985b) Dispersive ordering and monotone failure rate distributions. Adv. Appl. Prob. 17, 472-474.

DeshPande, J. AND Kochar, S. (1983) Dispersive ordering is the same as tail-ordering. $A d v$. Appl. Prob. 15, 686-687.

LEWIS, T. AND THOMPSON, J. (1981) Dispersive distributions and the connection between dispersivity and strong unimodality. J. Appl. Prob. 18, 76-90.

SATHE, Y. (1984) A comment on star-ordering and tail-ordering. Adv. Appl. Prob. 16, 692.

Shaked, M. (1982) Dispersive ordering of distributions. J. Appl. Prob. 19, 310-320. 